\title{
Eulerian polynomials via the Weyl algebra action
}

\author{
Jose Agapito ${ }^{2}$. Pasquale Petrullo ${ }^{1}$ D $\cdot$ Domenico Senato $^{1} \cdot$ Maria M. Torres ${ }^{2}$
}

Received: 17 March 2020 / Accepted: 16 November 2020 / Published online: 2 December 2020

(c) The Author(s) 2020

\begin{abstract}
Through the action of the Weyl algebra on the geometric series, we establish a generalization of the Worpitzky identity and new recursive formulae for a family of polynomials including the classical Eulerian polynomials. We obtain an extension of the Dobiński formula for the sum of rook numbers of a Young diagram by replacing the geometric series with the exponential series. Also, by replacing the derivative operator with the $q$-derivative operator, we extend these results to the $q$-analogue setting including the $q$-hit numbers. Finally, a combinatorial description and a proof of the symmetry of a family of polynomials introduced by one of the authors are provided.
\end{abstract}

Keywords Eulerian polynomials $\cdot$ Weyl algebra $\cdot$ Rook numbers $\cdot$ Permutation statistics $\cdot$ Formal power series

\section{Introduction}

This paper is mainly motivated by the idea of developing a theory for Eulerian polynomials and their generalizations through the formalism of the Weyl algebra. Our starting point is a family of polynomials, occasionally called hit polynomials [4,5], already covered in Riordan's book [16] in the late 1950s, and introduced by Kaplansky

Pasquale Petrullo

pasquale.petrullo@unibas.it

Jose Agapito

jaruiz@ciencias.ulisboa.pt

Domenico Senato

domenico.senato@unibas.it

Maria M. Torres

mmtorres@ciencias.ulisboa.pt

1 Dipartimento di Scienze Umane, Università degli Studi della Basilicata, Via Nazario Sauro 85, Potenza, Italy

2 Departamento de Matemática, Faculdade de Ciências, Universidade de Lisboa, 1749-016 Campo Grande, Lisboa, Portugal 
and Riordan [14]. Among other reasons, hit polynomials are interesting because of their combinatorial properties linked to rook numbers. Let us recall some notions and briefly describe the context. A non-attacking rook placement on a board $D$ is a set $P$ of boxes of $D$ with no two boxes in the same row or column. The number $r_{k}(D)$ of non-attacking rook placements $P$ on $D$ with $|P|=k$ is said to be the $k$-th rook number of $D$. If $D=D_{\lambda}$ is the Young diagram of a partition $\lambda$, then we write $r_{k}(\lambda)$ for the $k$-th rook number of $D_{\lambda}$. In particular, for the staircase partition $\delta_{n}:=(n, n-1, \ldots, 1)$, it is well-known that the rook numbers $r_{k}\left(\delta_{n-1}\right)$ are the Stirling numbers of the second kind $S(n, n-k)$. In this sense, the sum $R_{\lambda}=\sum_{k} r_{k}(\lambda)$ can be regarded as a generalized Bell number. By identifying the permutations in the symmetric group $\mathfrak{S}_{n}$ with the placements on the square diagram $D_{n}$ consisting of $n$ rows of length $n$, for any partition $\lambda$ such that $D_{\lambda} \subseteq D_{n}$, we set

$$
\mathcal{A}_{n, \lambda}(x):=\sum_{\sigma \in \mathfrak{S}_{n}} x^{\left|\sigma \cap D_{\lambda}\right|}
$$

The polynomials $\mathcal{A}_{n, \lambda}(x)$ often occur within the well developed literature on rook theory [4,6,9-14]. It is well-known that the classical Eulerian polynomials $A_{n}(x)$ arise as $\mathcal{A}_{n, \delta_{n-1}}(x)$. In Sect. 3, we will show that $\mathcal{A}_{n, \delta_{n-r}}(x)$ agrees with the polynomial ${ }^{r} A_{n}(x)$ introduced by Foata and Schützenberger [7]. This connection motivates a generalized notion of the excedance statistic that allows another combinatorial description of the polynomial $\mathcal{A}_{n, \lambda}(x)$. A classical formula of Frobenius, relating the Stirling numbers of the second kind and the Eulerian polynomials, extends in a straightforward manner to the following identity [4]

$$
\mathcal{A}_{n, \lambda}(x)=\sum_{k \geq 0} r_{k}(\lambda)(n-k) !(x-1)^{k} .
$$

Based on a $q$-analogue of rook numbers, Garsia and Remmel [8] provided a $q$-analogue for the polynomials $\mathcal{A}_{n, \lambda}(x)$ that generalizes identity (1). Dworkin [5] further studied the recursive properties of such polynomials and also gave a direct combinatorial interpretation of their coefficients, the $q$-hit numbers.

In the seventies, Navon [15] showed that rook placements also provide a natural combinatorial framework for the algebras generated by annihilation and creation operators, and in particular for the so-called normal ordering problem [2,3,17]. Recall that, if $\mathbf{X}$ denotes the operator of multiplication by $x$, and $\mathbf{D}=\frac{d}{d x}$ denotes the usual derivative operator, then $\mathbf{D X}-\mathbf{X D}=1$ and the algebra generated by $\mathbf{X}$ and $\mathbf{D}$ is referred to as the Weyl algebra. The normal ordering of any product $\boldsymbol{\Pi}$ involving $a$ occurrences of the operator $\mathbf{X}$ and $b$ occurrences of the operator $\mathbf{D}$ is given by

$$
\boldsymbol{\Pi}=\sum_{k \geq 0} r_{k}(\lambda) \mathbf{X}^{a-k} \mathbf{D}^{b-k}
$$

where $\lambda$ is a suitable partition associated with $\Pi$. In this setting, the Stirling numbers of the second kind arise as the normal ordering coefficients of $\Pi=(\mathbf{X D})^{n}$. 
We show that the polynomials $\mathcal{A}_{n, \lambda}(x)$ naturally describe the action of any product of the operators $\mathbf{D}$ and $\mathbf{X}$ on the geometric series $1 /(1-x)$. More precisely, given a partition $\lambda=\left(\lambda_{1}, \lambda_{2}, \ldots, \lambda_{l}\right)$, we define an operator $\Pi_{\lambda}$ such that for any square diagram $D_{n}$ containing $D_{\lambda}$,

$$
\boldsymbol{\Pi}_{\lambda} \mathbf{D}^{n-\lambda_{1}} \frac{1}{1-x}=\frac{\mathcal{A}_{n, \lambda(n)}(x)}{(1-x)^{n+1}},
$$

where $\lambda^{(n)}$ is a partition that we call the reduced complement of $\lambda$ in $D_{n}$ (Theorem 5). A first consequence of this point of view is that the polynomials of Garsia and Remmel arise when the operator $\boldsymbol{\Pi}_{\lambda, q} \mathbf{D}_{q}^{n-\lambda_{1}}$, obtained from $\boldsymbol{\Pi}_{\lambda} \mathbf{D}^{n-\lambda_{1}}$ by replacing $\mathbf{D}$ with the $q$-derivative $\mathbf{D}_{q}$, acts on $1 /(1-x)$. More precisely, they are the polynomials $\mathcal{A}_{n, \lambda}(x, q)$ such that

$$
\boldsymbol{\Pi}_{\lambda, q} \mathbf{D}_{q}^{n-\lambda_{1}} \frac{1}{1-x}=\frac{\mathcal{A}_{n, \lambda^{(n)}}(x, q)}{(1-x)(1-x q) \cdots\left(1-x q^{n}\right)} .
$$

In addition, straightforward manipulations of derivatives and formal power series allow us to establish a generalization of the classical Worpitzky identity (Corollary 6), a remarkably and seemingly new property of the polynomials $\mathcal{A}_{n, \lambda}(x)$ with respect to derivation (Corollary 7), and a recursion formula to compute $\mathcal{A}_{n, \lambda}(x)$ (Corollary 8). When $\lambda=\delta_{n-r}$ a new recursive formula relating the polynomials ${ }^{r} A_{n}(x)$ and the classical Eulerian polynomials is obtained. In turn, each of these results provide a corresponding $q$-analogue simply by replacing $\mathbf{D}$ with $\mathbf{D}_{q}$ (Corollaries 9,10,11). Furthermore, by letting $\Pi_{\lambda} \mathbf{D}^{n-\lambda_{1}}$ act on the formal power series expansion of $e^{x}$, we recover an extension of the classical Dobiński formula for the Bell numbers (identity (27)), and its $q$-analogue (identity (28)). Finally, we provide a combinatorial description and a proof of the symmetry property of the polynomials $A_{r, s, n}(x)$ (Proposition 13), defined by

$$
\left(\mathbf{X}^{r} \mathbf{D}^{s}\right)^{n} \frac{1}{1-x}=\frac{A_{r, s, n}(x)}{(1-x)^{s n+1}}
$$

and introduced by one of the authors of the present paper [1].

\section{Partitions and rook numbers}

By a partition, we mean a finite non-increasing vector $\lambda=\left(\lambda_{1}, \lambda_{2}, \ldots, \lambda_{l}\right)$ of positive integers called parts of $\lambda$. The number of parts of $\lambda$ is called the length of $\lambda$, and denoted by $\ell(\lambda)$. The Young diagram (or Ferrers board) of $\lambda$ is a left-aligned array of boxes, displayed in $\ell(\lambda)$ rows consisting of $\lambda_{1}, \lambda_{2}, \ldots, \lambda_{l}$ boxes, from top to bottom. In analogy with matrix notation, given a Young diagram $D$, we let $D_{i, j}$ denote the box of $D$ occurring at the $i$-th row (counting from top to bottom) and at the $j$-th column (counting from left to right). For instance, the Young diagram of $\lambda=(4,4,4,2,2,1)$ is shown in Fig. 1A, with a bullet drawn in the box $D_{3,2}$. The conjugate of $\lambda$ is the 
partition $\lambda^{\prime}$ whose diagram $D_{\lambda^{\prime}}$ is obtained by reflecting $D_{\lambda}$ with respect to its main diagonal. For example, the conjugate of $\lambda=(4,4,4,2,2,1)$ is $\lambda^{\prime}=(6,5,3,3)$ and its Young diagram is shown in Fig. 1B. The border of a Young diagram $D$ is by definition the subset of those sides lying at the rightmost position in a row, or at a lowest position in a column. The border of $D_{(4,4,4,2,2,1)}$ is highlighted in Fig. 1c.

Given any vectors $\boldsymbol{r}=\left(r_{1}, r_{2}, \ldots, r_{k}\right)$ and $\boldsymbol{u}=\left(u_{1}, u_{2}, \ldots, u_{k}\right)$ of positive integers, we let $\lambda_{\boldsymbol{r}, \boldsymbol{u}}$ denote the unique partition whose Young diagram has border with horizontal strips of lengths $r_{1}, r_{2}, \ldots, r_{k}$ (from left to right), and vertical strips of lengths $u_{1}, u_{2}, \ldots, u_{k}$ (from bottom to top). For instance, we have $\lambda_{(1,1,2),(1,2,3)}=$ $(4,4,4,2,2,1)$ as one may check from the horizontal and vertical strips in Fig. 2.

Given two partitions $\lambda$ and $\mu$, we write $\lambda \subseteq \mu$ to mean that $D_{\lambda} \subseteq D_{\mu}$. Moreover, we let $D_{n}$ denote the square Young diagram of $n$ rows, and for any partition $\lambda=$ $\left(\lambda_{1}, \lambda_{2}, \ldots, \lambda_{l}\right)$ such that $D_{\lambda} \subseteq D_{n}$, we call reduced complement of $\lambda$ in $D_{n}$ the partition $\lambda^{(n)}:=\left(n-\lambda_{l}, n-\lambda_{l-1}, \ldots, n-\lambda_{1}\right)$. In terms of Young diagrams, $D_{\lambda^{(n)}}$ is obtained from $D_{n}$ by removing the boxes of $D_{\lambda}$, deleting all the rows of $D_{n}$ lying below $D_{\lambda}$, then rotating by $180^{\circ}$. For instance, the reduced complement of $(2,2,1)$ in $D_{4}$ is $(3,2,2)$ and of $(6,6,3,3)$ in $D_{9}$ is $(6,6,3,3)$. They are obtained by rotating the white diagrams in Fig. 3.

A non attacking rook placement on a Young diagram $D$, simply placement from now on, is a set $P$ of blocks of $D$ with no two boxes occurring in the same row or column. The number of placements on $D_{\lambda}$ consisting of $k$ boxes, usually called the $k$-th rook number of $\lambda$, will be denoted by $r_{k}(\lambda)$. For instance, we have $r_{3}(4,3,1)=4$ and indeed the four placements of three boxes on $D_{(4,3,1)}$ are depicted in Fig. 4.

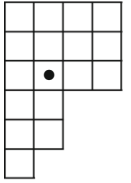

(a) $D_{\lambda}$

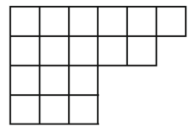

(b) $D_{\lambda^{\prime}}$

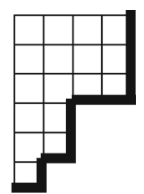

(c) The border of $D_{\lambda}$

Fig. 1 Young diagrams and their border

Fig. 2 Horizontal and vertical strips of a border
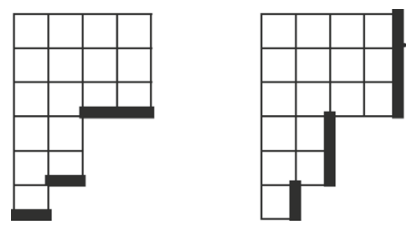

Fig. 3 The reduced complement (white boxes) of a partition (dark gray boxes)
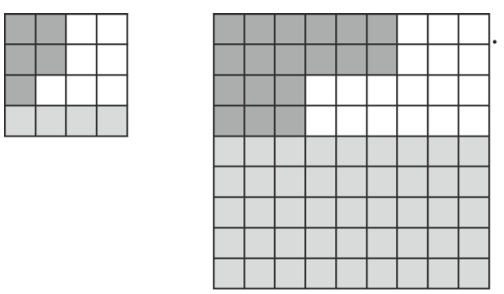

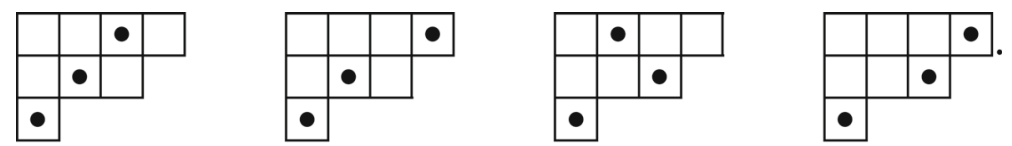

Fig. 4 A bullet is marked in each box of the placement

Fig. $5 D_{\lambda}$ in dark gray, $B_{\lambda}$ in light gray

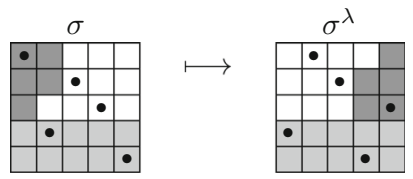

A placement of $n$ boxes on $D_{n}$ can be identified with a permutation matrix of order $n$. Thus, denoting the symmetric group of degree $n$ by $\mathfrak{S}_{n}$, we will consider the permutation $\sigma=\sigma_{1} \sigma_{2} \ldots \sigma_{n}$ and the placement $\left\{D_{1, \sigma(1)}, D_{2, \sigma(2)}, \ldots, D_{n, \sigma(n)}\right\}$ on $D=D_{n}$ as the same object. For instance, we identify the permutations 123, 132, $213,231,312,321$ in $\mathfrak{S}_{3}$ with the following placements on $D_{3}$ :

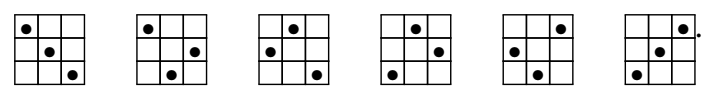

Note that $\sigma^{-1}$ is obtained by reflecting $\sigma$ in the main diagonal of $D_{n}$. Hence, for all $\sigma \in \mathfrak{S}_{n}$ and for all $\lambda$ such that $D_{\lambda} \subseteq D_{n}$ we have

$$
\left|\sigma \cap D_{\lambda}\right|=\left|\sigma^{-1} \cap D_{\lambda^{\prime}}\right|
$$

Moreover, given $\sigma \in \mathfrak{S}_{n}$, let $\sigma^{\lambda}=\sigma_{1}^{\lambda} \sigma_{2}^{\lambda} \ldots \sigma_{n}^{\lambda}$ be defined by

$$
\sigma_{i}^{\lambda}:= \begin{cases}n+1-\sigma_{\ell(\lambda)+1-i} & \text { if } 1 \leq i \leq \ell(\lambda) \\ n+1-\sigma_{n+1+\ell(\lambda)-i} & \text { if } \ell(\lambda)+1 \leq i \leq n\end{cases}
$$

It is easy to deduce that $\sigma \mapsto \sigma^{\lambda}$ is a bijective map. Now, set

$$
A_{\lambda}:=\left\{D_{i, j} \mid 1 \leq i \leq \ell(\lambda), 1 \leq j \leq n\right\} \text { and } B_{\lambda}:=D \backslash A_{\lambda}
$$

Observe that $\sigma^{\lambda}$ is obtained by separately rotating by $180^{\circ}$ the rectangles $A_{\lambda}$ and $B_{\lambda}$ (with respect to their center). For instance, let $\lambda=(2,2,1), n=5$ and $\sigma=13425$, then we have $\sigma^{\lambda}=23514$ as depicted in Fig. 5.

As $\left|\sigma \cap A_{\lambda}\right|=\ell(\lambda)$, we obtain

$$
\left|\sigma \cap D_{\lambda}\right|=\ell(\lambda)-\left|\sigma^{\lambda} \cap D_{\lambda^{(n)}}\right|
$$




\section{Generalized Eulerian polynomials}

Given a partition $\lambda$, and a positive integer $n$ such that $D_{\lambda} \subseteq D_{n}$, we define the polynomial $\mathcal{A}_{n, \lambda}(x)$ as follows:

$$
\mathcal{A}_{n, \lambda}(x):=\sum_{\sigma \in \mathfrak{S}_{n}} x^{\left|\sigma \cap D_{\lambda}\right|}
$$

Moreover, we set

$$
\mathcal{A}_{n, k, \lambda}:=\left|\left\{\sigma \in \mathfrak{S}_{n}:\left|\sigma \cap D_{\lambda}\right|=k\right\}\right|, \text { for } k=0,1, \ldots, n,
$$

and obtain

$$
\mathcal{A}_{n, \lambda}(x):=\sum_{k \geq 0} \mathcal{A}_{n, k, \lambda} x^{k}
$$

Example 1 Let $\lambda=(2,2,1)$ and $n=3$. In order to obtain $\mathcal{A}_{3,(2,2,1)}(x)$, we compute the cardinality of $\sigma \cap D_{\lambda}$, for each $\sigma \in \mathfrak{S}_{3}$.

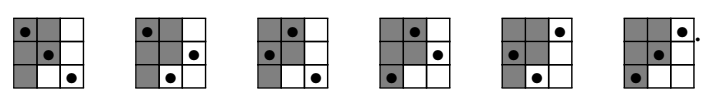

We get $\mathcal{A}_{3,(2,2,1)}(x)=4 x^{2}+2 x$. Note that by reflecting with respect to the main diagonal of $D_{3}$ (i.e., taking images under the bijection $\sigma \mapsto \sigma^{-1}$ ) one obtains $\mathcal{A}_{3,(3,2)}(x)=4 x^{2}+2 x=\mathcal{A}_{3, \lambda^{\prime}}(x)$,
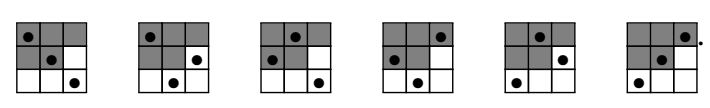

Proposition 1 Given a partition $\lambda$ and a positive integer $n$ such that $D_{\lambda} \subseteq D_{n}$, we have

(i) $\mathcal{A}_{n, \lambda}(1)=n !$

(ii) $\mathcal{A}_{n, \lambda^{\prime}}(x)=\mathcal{A}_{n, \lambda}(x)$;

(iii) $\mathcal{A}_{n, \lambda^{(n)}}(x)=x^{\ell(\lambda)} \mathcal{A}_{n, \lambda}(1 / x)$.

Proof From (5) and (2), we have (i) and (ii), respectively. Moreover, by means of $\sigma \mapsto \sigma^{\lambda}$ and (4) we have

$$
x^{\ell(\lambda)} \mathcal{A}_{n, \lambda}(1 / x)=\sum_{\sigma \in \mathfrak{S}_{n}} x^{\ell(\lambda)-\left|\sigma \cap D_{\lambda}\right|}=\sum_{\sigma \in \mathfrak{S}_{n}} x^{\left|\sigma^{\lambda} \cap D_{\lambda(n)}\right|}=\mathcal{A}_{n, \lambda(n)}(x),
$$

which gives (i). 
Note that (iii) means that the coefficients of $\mathcal{A}_{n, \lambda}(x)$, read in decreasing order of degree, agree with the coefficients of $\mathcal{A}_{n, \lambda^{(n)}}(x)$, read in increasing order of degree. For instance, if $\lambda=(3,3,2,1)$ then $\lambda^{(7)}=(6,5,4,4)$ and in fact we have

$$
\mathcal{A}_{7,(3,3,2,1)}(x)=192 x^{3}+1704 x^{2}+2496 x+648
$$

and

$$
\mathcal{A}_{7,(6,5,4,4)}(x)=648 x^{4}+2496 x^{3}+1704 x^{2}+192 x \text {. }
$$

In particular, the following symmetry property holds.

Corollary 2 Let $n$ be a positive integer and $\lambda$ a partition such that $D_{\lambda} \subseteq D_{n}$. If $\lambda^{(n)}=\lambda$ then

$$
\mathcal{A}_{n, \lambda}(x)=x^{\ell(\lambda)} \mathcal{A}_{n, \lambda}(1 / x)
$$

Moreover, if $\left(\lambda^{\prime}\right)^{(n)}=\lambda^{\prime}$ then

$$
\mathcal{A}_{n, \lambda}(x)=x^{\lambda_{1}} \mathcal{A}_{n, \lambda}(1 / x)
$$

Proof Identity (7) follows from $\lambda=\lambda^{(n)}$ and (iii). Identity (8) follows from (iii) taking into account that $\ell\left(\lambda^{\prime}\right)=\lambda_{1}$.

An explicit expansion of $\mathcal{A}_{n, \lambda}(x)$ in terms of the basis $\left\{(x-1)^{i} \mid i \geq 0\right\}$ has been known since [14], where it is proved by using the inclusion-exclusion principle. Here, we provide an alternative and explicit proof.

Theorem 3 Given a partition $\lambda$ and a positive integer $n$ such that $D_{\lambda} \subseteq D_{n}$, we have

$$
\mathcal{A}_{n, \lambda}(x)=\sum_{i \geq 0} r_{i}(\lambda)(n-i) !(x-1)^{i} .
$$

Proof By (5) we have

$$
\mathcal{A}_{n, \lambda}(x+1)=\sum_{\sigma \in \mathfrak{S}_{n}}(x+1)^{\left|\sigma \cap D_{\lambda}\right|}=\sum_{(\sigma, B) \in \text { Pairs }} x^{|B|},
$$

where Pairs denotes the set of all $(\sigma, B)$ such that $\sigma \in \mathfrak{S}_{n}$ and $B \subseteq\left(\sigma \cap D_{\lambda}\right)$. Note that for all $(\sigma, B) \in$ Pairs, $B$ is a placement on $D_{\lambda}$. Now, for any given placement $B_{0}$ on $D_{\lambda}$, let us count the pairs $(\sigma, B)$ such that $B=B_{0}$. Assume $\left|B_{0}\right|=i$ and consider the permutation $\sigma^{B_{0}}$ obtained by adding to $B_{0}$ the $n-i$ available boxes on the main diagonal of $D:=D_{n}$, that is

$$
\sigma^{B_{0}}:=B_{0} \cup\left\{D_{i, i} \mid D_{i, j} \notin B_{0} \text { for all } j=1,2, \ldots, n\right\} .
$$

Clearly $\left(\sigma^{B_{0}}, B_{0}\right) \in$ Pairs. Moreover, we obtain all the pairs of type $\left(\sigma, B_{0}\right)$ by permuting the $n-i$ columns of $D$ with no boxes in $\sigma^{B_{0}} \backslash B_{0}$. As there are $r_{i}(\lambda)$ placements 
$B$ on $D_{\lambda}$ with $|B|=i$, the number of pairs $(\sigma, B)$ such that $|B|=i$ is $r_{i}(\lambda)(n-i)$ !. We recover

$$
\mathcal{A}_{n, \lambda}(x+1)=\sum_{i \geq 0} r_{i}(\lambda)(n-i) ! x^{i}
$$

which gives (9) when $x$ is replaced by $x-1$.

Example 2 Let $r$ be a nonnegative integer. Following Foata and Schützenberger [7], we consider the polynomial

$$
{ }^{r} A_{n}(x):=\sum_{\sigma \in \mathfrak{S}_{n}} x^{\mathrm{exc}_{r}(\sigma)},
$$

where

$$
\operatorname{exc}_{r}(\sigma):=\left|\left\{i \mid 1 \leq i \leq n, \sigma_{i} \geq i+r\right\}\right|
$$

Clearly, ${ }^{1} A_{n}(x)$ is the classical Eulerian polynomial. Now, let $\sigma \mapsto \sigma^{\prime}$ denote the bijection defined on $\mathfrak{S}_{n+r}$ by $\sigma_{i}^{\prime}:=n+r+1-\sigma_{i}$, for $i=1,2, \ldots n+r$. Observe that $\sigma_{i} \leq n+1-i$ if and only if $\sigma_{i}^{\prime} \geq r+i$. As a consequence, we obtain

$$
\mathcal{A}_{n+r, \delta_{n}}(x)=\sum_{\sigma \in \mathfrak{S}_{n+r}} x^{\left|\sigma \cap D_{\delta_{n}}\right|}=\sum_{\sigma \in \mathfrak{S}_{n+r}} x^{\operatorname{exc}_{r}\left(\sigma^{\prime}\right)}={ }^{r} A_{n+r}(x),
$$

or equivalently ${ }^{r} A_{n}(x)=\mathcal{A}_{n, \delta_{n-r}}(x)$. From (9), we recover the following Frobenius identity for the polynomials ${ }^{r} A_{n}(x)$ [7]:

$$
{ }^{r} A_{n}(x)=\sum_{k \geq 0} S(n+1-r, n+1-r-k)(n-k) !(x-1)^{k} .
$$

The following generalization of the notion of excedance is motivated by Example 2 . Given a partition $\lambda=\left(\lambda_{1}, \lambda_{2}, \ldots, \lambda_{l}\right)$, a positive integer $n$ such that $D_{\lambda} \subseteq D_{n}$, and a permutation $\sigma=\sigma_{1} \sigma_{2} \ldots \sigma_{n} \in \mathfrak{S}_{n}$, we set

$$
\operatorname{exc}_{\lambda}(\sigma):=\left|\left\{i \mid 1 \leq i \leq n, \sigma_{i}>n+1-\lambda_{i}\right\}\right|
$$

where $\lambda_{i}=0$ is assumed for $\ell(\lambda)<i \leq n$. As before, the complement bijection $\sigma \mapsto \sigma^{\prime}$ provides

$$
\left|\sigma \cap D_{\lambda}\right|=\operatorname{exc}_{\lambda}\left(\sigma^{\prime}\right)
$$

so that we get

$$
\mathcal{A}_{n, \lambda}(x)=\sum_{\sigma \in \mathfrak{S}_{n}} x^{\operatorname{exc}_{\lambda}(\sigma)}
$$




\section{The Weyl algebra action}

Let $\mathbf{D}, \mathbf{X}: \mathbb{Z}[x] \rightarrow \mathbb{Z}[x]$ denote the derivative operator and the operator of multiplication by $x$, respectively. As $\mathbf{D X}-\mathbf{X D}=1$ the following normal ordering problem may be posed: given any product $\boldsymbol{\Pi}$ involving $a$ occurrences of the operator $\mathbf{D}$ and $b$ occurrences of the operator $\mathbf{X}$, find the coefficients $c_{i}(\boldsymbol{\Pi})$ satisfying

$$
\boldsymbol{\Pi}=\sum_{i \geq 0} c_{i}(\boldsymbol{\Pi}) \mathbf{X}^{b-i} \mathbf{D}^{a-i}
$$

A beautiful answer to this problem was given by Navon [15] in terms of placements on Young diagrams. Here, we recast Navon's result following the work of Varvak [17]. For any partition $\lambda$, we set

$$
\Pi_{\lambda}:=\mathbf{D}^{r_{1}} \mathbf{X}^{u_{1}} \mathbf{D}^{r_{2}} \mathbf{X}^{u_{2}} \ldots \mathbf{D}^{r_{k}} \mathbf{X}^{u_{k}}
$$

where $\boldsymbol{r}=\left(r_{1}, r_{2}, \ldots, r_{k}\right)$ and $\boldsymbol{u}=\left(u_{1}, u_{2}, \ldots, u_{k}\right)$ are the unique vectors satisfying $\lambda=\lambda_{\boldsymbol{r}, \boldsymbol{u}}$. Note that $\lambda_{1}=r_{1}+r_{2}+\cdots+r_{k}$ and $\ell(\lambda)=u_{1}+u_{2}+\cdots+u_{k}$.

Theorem 4 For any partition $\lambda$, we have

$$
\boldsymbol{\Pi}_{\lambda}=\sum_{i \geq 0} r_{i}(\lambda) \mathbf{X}^{\ell(\lambda)-i} \mathbf{D}^{\lambda_{1}-i}
$$

Proof Let $\lambda=\left(\lambda_{1}, \lambda_{2}, \ldots, \lambda_{l}\right)$. A straightforward computation shows that $\Pi_{\lambda} 1=$ $r_{\lambda_{1}}(\lambda) x^{\ell(\lambda)-\lambda_{1}}$. Set

$$
\mu:=(\underbrace{\lambda_{1}, \lambda_{1}, \ldots, \lambda_{1}}_{m+1}, \lambda_{2}, \ldots, \lambda_{l}) \text { and } \mu \backslash \lambda:=(\underbrace{\lambda_{1}, \lambda_{1}, \ldots, \lambda_{1}}_{m}) .
$$

It follows that $\boldsymbol{\Pi}_{\lambda} x^{m}=\boldsymbol{\Pi}_{\lambda} \mathbf{X}^{m} 1=\Pi_{\mu} 1=r_{\lambda_{1}}(\mu) x^{m+\ell(\lambda)-\lambda_{1}}$. On the other hand, we may compute $r_{\lambda_{1}}(\mu)$ in the following alternative way,

$$
r_{\lambda_{1}}(\mu)=\sum_{k \geq 0} r_{k}(\lambda) r_{\lambda_{1}-i}(\mu \backslash \lambda)=\sum_{i \geq 0} r_{i}(\lambda) \frac{m !}{\left(m-\lambda_{1}-i\right) !} .
$$

Then, we conclude

$$
\sum_{i \geq 0} r_{i}(\lambda) \mathbf{X}^{\ell(\lambda)-i} \mathbf{D}^{\lambda_{1}-i} x^{m}=r_{\lambda_{1}}(\mu) x^{m+\ell(\lambda)-\lambda_{1}}=\boldsymbol{\Pi}_{\lambda} x^{m} .
$$

The following theorem makes explicit the connection between the Weyl algebra and the polynomials $\mathcal{A}_{n, \lambda}(x)$. 
Theorem 5 For any partition $\lambda$ and any positive integer $n$ such that $D_{\lambda} \subseteq D_{n}$, we have

$$
\boldsymbol{\Pi}_{\lambda} \mathbf{D}^{n-\lambda_{1}} \frac{1}{1-x}=\frac{\mathcal{A}_{n, \lambda^{(n)}}(x)}{(1-x)^{n+1}} .
$$

Proof By (14) we obtain

$$
\begin{aligned}
\boldsymbol{\Pi}_{\lambda} \mathbf{D}^{n-\lambda_{1}} \frac{1}{1-x} & =\sum_{i \geq 0} r_{i}(\lambda) \mathbf{X}^{\ell(\lambda)-i} \mathbf{D}^{n-i} \frac{1}{1-x} \\
& =\sum_{i \geq 0} r_{i}(\lambda)(n-i) ! \frac{x^{\ell(\lambda)-i}}{(1-x)^{n-i+1}}
\end{aligned}
$$

hence

$$
(1-x)^{n+1} \boldsymbol{\Pi}_{\lambda} \mathbf{D}^{n-\lambda_{1}} \frac{1}{1-x}=\sum_{i \geq 0} r_{i}(\lambda)(n-i) ! x^{\ell(\lambda)-i}(1-x)^{i}
$$

Moreover, by (9) we have

$$
x^{\ell(\lambda)} \mathcal{A}_{n, \lambda}(1 / x)=\sum_{i \geq 0} r_{i}(\lambda)(n-i) ! x^{\ell(\lambda)-i}(1-x)^{i} .
$$

Finally, by comparing (17), (16) and Proposition 1 (iii), we have

$$
\frac{\mathcal{A}_{n, \lambda^{(n)}}(x)}{(1-x)^{n+1}}=\frac{x^{\ell(\lambda)} \mathcal{A}_{n, \lambda}(1 / x)}{(1-x)^{n+1}}=\Pi_{\lambda} \mathbf{D}^{n-\lambda_{1}} \frac{1}{1-x} .
$$

A first consequence of (15) is the following extension of the Worpitzky identity for Eulerian polynomials.

Corollary 6 Let $m$ be a positive integer. For any partition $\lambda=\left(\lambda_{1}, \lambda_{2}, \ldots, \lambda_{l}\right)$ and any positive integer $n$ such that $D_{\lambda} \subseteq D_{n}$, we have

$$
\prod_{i=0}^{n-1}\left(m+\lambda_{n-i}^{\prime}-i\right)=\sum_{k \geq 0}\left(\begin{array}{c}
m+\ell(\lambda)-k \\
n
\end{array}\right) \mathcal{A}_{n, k, \lambda^{(n)}},
$$

where $\lambda^{\prime}=\left(\lambda_{1}^{\prime}, \lambda_{2}^{\prime}, \ldots, \lambda_{l^{\prime}}^{\prime}\right)$ is the conjugate of $\lambda$ and we assume that $\lambda_{i}^{\prime}=0$ for $i>l^{\prime}=\lambda_{1}$.

\section{Proof Set}

$$
\mu:=(\underbrace{n, n, \ldots, n}_{m}, \lambda_{1}, \lambda_{2}, \ldots, \lambda_{l})
$$


and observe that

$$
r_{n}(\mu)=\prod_{i=0}^{n-1}\left(m+\lambda_{n-i}^{\prime}-i\right)
$$

Moreover, we have $\Pi_{\lambda} \mathbf{D}^{n-\lambda_{1}} x^{m}=\Pi_{\mu} 1=r_{n}(\mu) x^{m+\ell(\lambda)-n}$ and then the left-hand side of (15) is given by

$$
\boldsymbol{\Pi}_{\lambda} \mathbf{D}^{n-\lambda_{1}} \frac{1}{1-x}=\sum_{m \geq 0} \prod_{i=0}^{n-1}\left(m+\lambda_{n-i}^{\prime}-i\right) x^{m+\ell(\lambda)-n}
$$

From (6), the right-hand side of (15) may be rewritten as

$$
\sum_{i \geq 0}\left(\sum_{k \geq 0}\left(\begin{array}{c}
n+i-k \\
n
\end{array}\right) \mathcal{A}_{n, k, \lambda(n)}\right) x^{i}
$$

Hence, (18) follows by extracting the coefficient of $x^{m-n+\ell(\lambda)}$ from both sides in (15).

Example 3 Setting $\lambda=(n-1, n-2, \ldots, r)$ in (18), and observing that $\lambda^{(n)}=\delta_{n-r}$, we obtain the following Worpitzky identity [7],

$$
m^{n-r} \frac{m !}{(m-r) !}=\sum_{k \geq 0}\left(\begin{array}{c}
m+r-k \\
n
\end{array}\right){ }^{r} A_{n, k} .
$$

Of course, $r=1$ leads to the Worpitzky identity for Eulerian numbers:

$$
m^{n}=\sum_{k \geq 0}\left(\begin{array}{c}
m+1-k \\
n
\end{array}\right) A_{n, k}
$$

A further consequence of (15) is a remarkable property of the polynomials $\mathcal{A}_{n, \lambda}(x)$ with respect to derivation. In terms of the underlined Young diagrams, this property encodes the evolution of the polynomials $\mathcal{A}_{n, \lambda}(x)$, for a fixed partition $\lambda$, with respect to square diagrams $D_{n}$ of increasing size.

Corollary 7 For any partition $\lambda$ and any positive integer $n$ such that $D_{\lambda} \subseteq D_{n}$, we have

$$
\mathbf{D} \frac{\mathcal{A}_{n, \lambda}(x)}{(1-x)^{n+1}}=\frac{\mathcal{A}_{n+1, \lambda}(x)}{(1-x)^{n+2}} \text {. }
$$

Proof If $\lambda=\left(\lambda_{1}, \lambda_{2}, \ldots, \lambda_{l}\right)$ then we set $\lambda+1:=\left(\lambda_{1}+1, \lambda_{2}+1, \ldots, \lambda_{l}+1\right)$. Note that the reduced complements of $\lambda$ in $D_{n}$ and of $\lambda+1$ in $D_{n+1}$ agree, hence from (15) 
we have

$\mathbf{D} \frac{\mathcal{A}_{n, \lambda(n)}(x)}{(1-x)^{n+1}}=\mathbf{D} \boldsymbol{\Pi}_{\lambda} \mathbf{D}^{n-\lambda_{1}} \frac{1}{1-x}=\boldsymbol{\Pi}_{\lambda+1} \mathbf{D}^{(n+1)-\left(\lambda_{1}+1\right)} \frac{1}{1-x}=\frac{\mathcal{A}_{n+1, \lambda^{(n)}}(x)}{(1-x)^{n+2}}$.

Identity (19) suggests that the polynomials $\mathcal{A}_{n, \lambda}(x)$ indexed by the smallest $n$ such that $D_{\lambda} \subseteq D_{n}$, play a special role. Indeed, for any partition $\lambda$, we set

$$
n(\lambda):=\max \left\{\lambda_{1}, \ell(\lambda)\right\}
$$

and define

$$
\mathcal{A}_{\lambda}(x):=\mathcal{A}_{n(\lambda), \lambda}(x)
$$

Hence, we obtain the following recursive rule.

Corollary 8 For any partition $\lambda$ and any positive integer $n$ such that $D_{\lambda} \subseteq D_{n}$, we have

$$
\mathcal{A}_{n, \lambda}(x)=(1-x)^{n+1} \mathbf{D}^{n-n(\lambda)} \frac{\mathcal{A}_{\lambda}(x)}{(1-x)^{n(\lambda)+1}} .
$$

Proof Identity (22) follows by iterating (19).

Remark 1 Note that, by Proposition 1 (iii) and (7) we have $\mathcal{A}_{\delta_{n}}(x)=x A_{n}(x)$. Therefore, by setting $\lambda=\delta_{n-r}$ in (22), the polynomials ${ }^{r} A_{n}(x)$ are obtained via suitable derivatives involving the classical Eulerian polynomials,

$$
{ }^{r} A_{n}(x)=(1-x)^{n+1} \mathbf{D}^{r} \frac{x A_{n-r}(x)}{(1-x)^{n-r+1}} .
$$

\section{$5 q$-analogues arising from the $q$-Weyl algebra}

Let $\mathbf{D}_{q}$ denote the $q$-derivative operator acting on the polynomial $p(x)$ according to the following rule,

$$
\mathbf{D}_{q} p(x)=\frac{p(q x)-p(x)}{q x-x} .
$$

We have $\mathbf{D}_{q} \mathbf{X}-q \mathbf{X} \mathbf{D}_{q}=1$ and the algebra generated by $\mathbf{X}, \mathbf{D}_{q}$ is a $q$-analogue of the Weyl algebra. Now, let $[i]:=1+q+\cdots+q^{i-1}$ denote the $q$-integer, and for all partitions $\lambda$, let $\boldsymbol{\Pi}_{\lambda, q}$ be obtained from (13) by replacing $\mathbf{D}$ with $\mathbf{D}_{q}$. As $\mathbf{D}_{q}^{i} x^{m}=$ $[m][m-1] \cdots[m-i+1] x^{m-i}$, straightforward computations show that

$$
\boldsymbol{\Pi}_{\lambda, q} \mathbf{D}_{q}^{n-\lambda_{1}} \frac{1}{1-x}=\sum_{m \geq 0} \prod_{i=0}^{n-1}\left[m+\lambda_{n-i}^{\prime}-i\right] x^{m-n+\ell(\lambda)} \text {. }
$$


Note that the right-hand side of (23) agrees with the right-hand side of identity (I.11) in the paper of Garsia and Remmel [8], as can be seen by setting $a_{i+1}=n-\ell(\lambda)+\lambda_{n-i}^{\prime}$ for $0 \leq i \leq n-1$, that is by setting $\lambda=\mu^{(n)}$ for $\mu:=\left(a_{n}, a_{n-1}, \ldots, a_{1}\right)$. Now, we let $\mathcal{A}_{n, \lambda^{(n)}}(x, q)$ denote the polynomial defined by

$$
\boldsymbol{\Pi}_{\lambda, q} \mathbf{D}_{q}^{n-\lambda_{1}} \frac{1}{1-x}=\frac{\mathcal{A}_{n, \lambda^{(n)}}(x, q)}{(1-x)(1-x q) \cdots\left(1-x q^{n}\right)},
$$

and the right-hand side of (I.12) in [8] ensures that $Q_{A}(x, q)=\mathcal{A}_{n, \lambda^{(n)}}(x, q)$ when the partition $\lambda$ is chosen such that $a_{i+1}=n-\ell(\lambda)+\lambda_{n-i}^{\prime}$ for $0 \leq i \leq n-1$. First, we recall that

$$
\frac{1}{(1-x)(1-x q) \cdots\left(1-x q^{n}\right)}=\sum_{k \geq 0}\left[\begin{array}{c}
n+k \\
n
\end{array}\right] x^{k}
$$

Moreover, we define $\mathcal{A}_{n, k, \lambda^{(n)}}(q)$ by

$$
\mathcal{A}_{n, \lambda^{(n)}}(x, q)=\sum_{k \geq 0} \mathcal{A}_{n, k, \lambda^{(n)}}(q) x^{k}
$$

and compare the coefficients of (23) and (24) to obtain the following $q$-analogue of Corollary 6.

Corollary 9 Let $m$ be a positive integer. For any partition $\lambda=\left(\lambda_{1}, \lambda_{2}, \ldots, \lambda_{l}\right)$ and any positive integer $n$ such that $D_{\lambda} \subseteq D_{n}$, we have

$$
\prod_{i=0}^{n-1}\left[m+\lambda_{n-i}^{\prime}-i\right]=\sum_{k \geq 0}\left[\begin{array}{c}
m+\ell(\lambda)-k \\
n
\end{array}\right] \mathcal{A}_{n, k, \lambda(n)}(q),
$$

where $\lambda^{\prime}=\left(\lambda_{1}^{\prime}, \lambda_{2}^{\prime}, \ldots, \lambda_{l^{\prime}}^{\prime}\right)$ is the conjugate of $\lambda$ and we assume that $\lambda_{i}^{\prime}=0$ for $i>l^{\prime}=\lambda_{1}$.

Moreover, simply by replacing $\mathbf{D}$ with $\mathbf{D}_{q}$ in the proof of Corollary 7 , we obtain the following $q$-analogue of (19).

Corollary 10 For any partition $\lambda=\left(\lambda_{1}, \lambda_{2}, \ldots, \lambda_{l}\right)$ and any positive integer $n$ such that $D_{\lambda} \subseteq D_{n}$, we have

$$
\mathbf{D}_{q} \frac{\mathcal{A}_{n, \lambda}(x, q)}{(1-x)(1-x q) \cdots\left(1-x q^{n}\right)}=\frac{\mathcal{A}_{n+1, \lambda}(x, q)}{(1-x)(1-x q) \cdots\left(1-x q^{n+1}\right)} .
$$

We let $\mathcal{A}_{\lambda}(x, q):=\mathcal{A}_{n(\lambda), \lambda}(x, q)$ and easily obtain the $q$-analogue of the recursive property (22). 
Corollary 11 For any partition $\lambda=\left(\lambda_{1}, \lambda_{2}, \ldots, \lambda_{l}\right)$ and any positive integer $n$ such that $D_{\lambda} \subseteq D_{n}$, we have

$$
\mathcal{A}_{n+1, \lambda}(x, q)=(1-x)(1-x q) \cdots\left(1-x q^{n+1}\right) \mathbf{D}_{q}^{n-n(\lambda)} \frac{\mathcal{A}_{\lambda}(x, q)}{(1-x)(1-x q) \cdots\left(1-x q^{n(\lambda)}\right)} .
$$

We explicitly remark that the polynomials $\mathcal{A}_{n, k, \lambda}(q)$ are the so-called $q$-hit numbers $[5]$.

\section{Further generalizations and applications}

\subsection{An application to the operator $\left(X^{r} D^{s}\right)^{n}$}

We now consider the polynomials $A_{r, s, n}(x)$ introduced in [1] and defined by

$$
\left(\mathbf{X}^{r} \mathbf{D}^{s}\right)^{n} \frac{1}{1-x}=\frac{A_{r, s, n}(x)}{(1-x)^{s n+1}},
$$

for all positive integers $r \leq s$ and $n \geq 1$. Let $\boldsymbol{r}=\left(r_{1}, r_{2}, \ldots, r_{n}\right)$ and $\boldsymbol{u}=$ $\left(u_{1}, u_{2}, \ldots, u_{n}\right)$ satisfy $r_{1}=r_{2}=\ldots=r_{n}=s$ and $u_{1}=u_{2}=\ldots=u_{n}=r$, set $\delta_{r, s, n}:=\lambda_{\boldsymbol{r}, \boldsymbol{u}}$. The Young diagram of $\delta_{r, s, n}$ is obtained from $D_{\delta_{n}}$ by replacing each box in $D_{\delta_{n}}$ with a rectangular diagram of $s$ columns and $r$ rows. For example, the Young diagram of $\delta_{2,3,2}$ is $D_{(6,6,3,3)}$, as shown in Fig. 3 (dark gray) as a subset of $D_{9}$. We denote by $\operatorname{exc}_{r, s, n}$ the deformation of the excedance statistic induced by $\lambda=\delta_{r, s, n}$ via (11). In particular, for all $\sigma \in \mathfrak{S}_{s n}$, we have

$$
\left.\operatorname{exc}_{r, s, n-1}(\sigma)=\mid\left\{i=\left(i_{1}-1\right) r+i_{2} \mid 1 \leq i_{1} \leq n-1,1 \leq i_{2} \leq r, \sigma_{i}>s i_{1}\right)\right\} \mid \text {. }
$$

Note that, as $\delta_{1,1, n-1}=\delta_{n-1}$ (by convention $\delta_{0}=(1)$ ), we have $\operatorname{exc}_{1,1, n-1}(\sigma)=$ $\operatorname{exc}(\sigma)$ for all $\sigma \in \mathfrak{S}_{n}$. The following result gives a combinatorial explanation for the identity $A_{r, s, n}(1)=(s n)$ ! [1].

Proposition 12 For all positive integers $r \leq s$ and $n \geq 1$, we have

$$
A_{r, s, n}(x)=x^{r} \mathcal{A}_{s n, \delta_{r, s, n-1}}(x)=x^{r} \sum_{\sigma \in \mathfrak{S}_{s n}} x^{\operatorname{exc}_{r, s, n-1}(\sigma)}
$$

Proof Let $\lambda:=\delta_{s, r, n-1}$. From

$$
\left(\mathbf{X}^{r} \mathbf{D}^{s}\right)^{n}=\mathbf{X}^{r}\left(\mathbf{D}^{s} \mathbf{X}^{r}\right)^{n-1} \mathbf{D}^{s}=\mathbf{X}^{r} \boldsymbol{\Pi}_{\lambda} \mathbf{D}^{s n-s(n-1)},
$$

by virtue of Theorem 5 we obtain

$$
\frac{A_{r, s, n}(x)}{(1-x)^{s n+1}}=\mathbf{X}^{r} \boldsymbol{\Pi}_{\lambda} \mathbf{D}^{s n-s(n-1)} \frac{1}{1-x}=\frac{x^{r} \mathcal{A}_{s n, \lambda^{(s n)}}(x)}{(1-x)^{s n+1}} .
$$


As $\delta_{r, s, n-1}=\delta_{r, s, n-1}^{(s n)}$,

$$
A_{r, s, n}(x)=x^{r} \mathcal{A}_{s n, \delta_{r, s, n-1}}(x),
$$

and via (12) we deduce (26).

Now, we prove the following result originally conjectured in [1].

Proposition 13 For all positive integers $r \leq s$ and $n \geq 1$, we have

$$
A_{r, s, n}(x)=x^{r(n-1)} A_{r, s, n}(1 / x) .
$$

Proof By taking into account Proposition 1(iii), as $\delta_{r, s, n-1}=\delta_{r, s, n-1}^{(s n)}$, and since $\ell\left(\delta_{r, s, n-1}\right)=r(n-1)$, from $(26)$, we have

$$
x^{r(n-1)} A_{r, s, n}(1 / x)=x^{r} x^{\ell\left(\delta_{r, s, n-1}\right)} \mathcal{A}_{s n, \delta_{r, s, n-1}}(1 / x)=A_{r, s, n}(x) .
$$

\subsection{Generalizations of the Dobiński formula}

One may think to replace the geometric series $1 /(1-x)$ in (15) and let any product $\Pi$ act on an arbitrary power series $f(x)$. More interestingly, one may look for those series $f(x)$ such that $\Pi f(x)$ has some combinatorial interest. Let us discuss the case $f(x)=e^{x}$, which leads to an extension of the Dobiński formula. Indeed, by (14) one obtains

$$
\boldsymbol{\Pi}_{\lambda} \mathbf{D}^{n-\lambda_{1}} e^{x}=e^{x} \sum_{k \geq 0} r_{k}(\lambda) x^{\ell(\lambda)-k}=e^{x} x^{\ell(\lambda)} R_{\lambda}(1 / x),
$$

where $R_{\lambda}(x)=\sum_{k} r_{k}(\lambda) x^{k}$ is the well-known rook polynomial associated with $D_{\lambda}$. On the other hand, by expanding $e^{x}$ we also have

$$
\boldsymbol{\Pi}_{\lambda} \mathbf{D}^{n-\lambda_{1}} e^{x}=\sum_{m \geq 0} \prod_{i=0}^{n-1}\left(m+\lambda_{n-i}^{\prime}-i\right) \frac{x^{m-n+\ell(\lambda)}}{m !},
$$

and then

$$
\sum_{m \geq 0} \prod_{i=0}^{n-1}\left(m+\lambda_{n-i}^{\prime}-i\right) \frac{x^{m-n+\ell(\lambda)}}{m !}=e^{x} x^{\ell(\lambda)} R_{\lambda}(1 / x) .
$$

Setting $x=1$ and $R_{\lambda}:=R_{\lambda}(1)$ we obtain the following generalization of the Dobiński formula

$$
\sum_{m \geq 0} \frac{\prod_{i=0}^{n-1}\left(m+\lambda_{n-i}^{\prime}-i\right)}{m !}=e R_{\lambda}
$$


The classical case arises when $\lambda=\delta_{n-1}$, and then $R_{\delta_{n-1}}=B_{n}$ is the $n$-th Bell number,

$$
\sum_{m \geq 0} \frac{m^{n}}{m !}=e B_{n}
$$

Moreover, replacing $n$ with $s n$, setting $\lambda=\delta_{r, s, n-1}$ and $B_{r, s, n}:=R_{\delta_{r, s, n-1}}$, we get a Dobiński formula for the sum of all generalized Stirling numbers $S_{r, s}(n, k):=$ $r_{s n-k}\left(\delta_{r, s, n-1}\right)[2]$,

$$
\sum_{m \geq 0} \frac{1}{(m-(s-r) n) !} \prod_{i=1}^{n} \frac{(m-(s-r) i) !}{(m-(s-r) i-r) !}=e B_{r, s, n} .
$$

In particular, when $r=s$, we recover

$$
\sum_{m \geq 0} \frac{1}{m !} \frac{m !^{n}}{(m-r) !^{n}}=e B_{r, r, n}
$$

In closing, to recover a $q$-analogue of (27), set

$$
\varepsilon(x):=\sum_{k \geq 0} \frac{x^{k}}{[k] !}
$$

where $[k] !:=[1][2] \cdots[k]$, and observe that $\mathbf{D}_{q} \varepsilon(x)=\varepsilon(x)$. We deduce

$$
\boldsymbol{\Pi}_{\lambda, q} \mathbf{D}_{q}^{n-\lambda_{1}} \varepsilon(x)=\varepsilon(x) \sum_{k \geq 0} r_{k}(\lambda, q) x^{\ell(\lambda)-k}=\varepsilon(x) x^{\ell(\lambda)} R_{\lambda}(1 / x, q),
$$

where $R_{\lambda}(x, q)=\sum_{k} r_{k}(\lambda, q) x^{\ell(\lambda)-k}$, and the $r_{k}(\lambda, q)$ are the $q$-rook numbers arising here as the normal ordering coefficients of $\boldsymbol{\Pi}_{\lambda, q} \mathbf{D}_{q}^{n-\lambda_{1}}$ (Theorem 6.1 in [17]). Finally, we set $\varepsilon:=\varepsilon(1)$ and $R_{\lambda}(q):=R_{\lambda}(1, q)$ and obtain the following result.

Proposition 14 For any partition $\lambda=\left(\lambda_{1}, \lambda_{2}, \ldots, \lambda_{l}\right)$ such that $D_{\lambda} \subseteq D_{n}$, we have

$$
\sum_{m \geq 0} \frac{\prod_{i=0}^{n-1}\left[m+\lambda_{n-i}^{\prime}-i\right]}{[m] !}=\varepsilon R_{\lambda}(q)
$$

where $\lambda^{\prime}=\left(\lambda_{1}^{\prime}, \lambda_{2}^{\prime}, \ldots, \lambda_{l^{\prime}}^{\prime}\right)$ is the conjugate of $\lambda$ and we assume that $\lambda_{i}^{\prime}=0$ for $i>l^{\prime}=\lambda_{1}$.

Acknowledgements The first and the fourth authors were partially supported by FCT-Fundação para a Ciência e a Tecnologia (Portugal), through the project UIDB/04721/2020.

Funding Open access funding provided by University degli Studi della Basilicata within the CRUI-CARE Agreement. 
Open Access This article is licensed under a Creative Commons Attribution 4.0 International License, which permits use, sharing, adaptation, distribution and reproduction in any medium or format, as long as you give appropriate credit to the original author(s) and the source, provide a link to the Creative Commons licence, and indicate if changes were made. The images or other third party material in this article are included in the article's Creative Commons licence, unless indicated otherwise in a credit line to the material. If material is not included in the article's Creative Commons licence and your intended use is not permitted by statutory regulation or exceeds the permitted use, you will need to obtain permission directly from the copyright holder. To view a copy of this licence, visit http://creativecommons.org/licenses/by/4.0/.

\section{References}

1. Agapito, J.: On symmetric polynomials with only real zeros and nonnegative $\gamma$-vectors. Linear Algebra Appl. 451, 260-289 (2014)

2. Blasiak, P.: Combinatorics of boson normal ordering and some applications. Concepts Phys. 1, 177-278 (2004)

3. Blasiak, P., Flajolet, P.: Combinatorial models of creation-annihilation. Sém. Lothar. Combin. 65, Article B65c (2011)

4. Butler, F., Can, M., Haglund, J., Remmel, J.B.: Rook Theory Notes, preprint, 2020. http://www.math. ucsd.edu/ remmel/

5. Dworkin, M.: An interpretation for Garsia and Remmel q-hit numbers. J. Combin. Theory Ser. A 81, 149-175 (1998)

6. Foata, D., Schützenberger, M.-P.: On the rook polynomials of Ferrers relations. In: Combinatorial Theory and its Applications, II (Proc. Colloq., Balatonfüred, 1969), vol. 2, pp. 413-436 (1970)

7. Foata, D., Schützenberger, M.-P.: Théorie Géométrique des Polynômes Eulériens. Lectures Notes in Mathematics, vol. 138. Springer, Berlin (1970)

8. Garsia, A.M., Remmel, J.B.: Q-Counting rook configurations and a formula of Frobenius. J. Combin. Theory Ser. A 41, 246-275 (1986)

9. Goldman, J.R., Joichi, J.T., Reiner, D.L., White, D.E.: Rook theory II: Boards of binomial type. SIAM J. Appl. Math. 31(4), 618-633 (1976)

10. Goldman, J.R., Joichi, J.T., White, D.E.: Rook theory I. Rook equivalence of Ferrers boards. Proc. Amer. Math. Soc. 52, 485-492 (1975)

11. Goldman, J.R., Joichi, J.T., White, D.E.: Rook theory III. Rook polynomials and the chormatic structure of graphs. J. Combin. Theory Ser. B 25, 135-142 (1978)

12. Goldman, J.R., Joichi, J.T., White, D.E.: Rook Theory IV. Orthogonal sequences of rook polynomials. Stud. Appl. Math. 56(3), 267-272 (1977)

13. Goldman, J.R., Joichi, J.T., White, D.E.: Rook Theory V. Rook polynomials, Möbius inversion and the umbral calculus. J. Combin. Theory Ser. A 21(2), 230-239 (1976)

14. Kaplansky, I., Riordan, J.: The problem of the rooks and its applications. Duke Math. J. 13, 259-268 (1946)

15. Navon, A.M.: Combinatorics and fermion algebra. Nuovo Cimento Soc. Ital. Fis. B 16, 324-330 (1973)

16. Riordan, J.: Introduction to Combinatorial Analysis. Wiley, New York (1958)

17. Varvak, A.: Rook numbers and the normal ordering problem. J. Combin. Theory Ser. A 112, 292-307 (2005)

Publisher's Note Springer Nature remains neutral with regard to jurisdictional claims in published maps and institutional affiliations. 